\title{
RY SGR: PULSATION RELATED PHENOMENON
}

\author{
W. A. Lawson \\ Mount John University Observatory \\ Dept. of Physics, Univ. of Canterbury \\ Christchurch \\ New Zealand
}

The absorption line splitting reported by Cottre11 and Lambert has been shown to be associated with the semi-regular pulsations. The event has a time scale of about 10 days ( 0.23 of a period) occuring about the radial velocity reversal at minimum radius. The velocity reversal is more rapid than indicated in an earlier radial velocity analysis by Alexander et al. There is evidence that the phase of the line splitting event is similar to the phase of the onset of the obscurational light declines.

\section{INTRODUCTION}

The low amplitude periodic variations of RY Sgr were first noted by Jacchia (1933). Jacchia found a 39 day period with an amplitude of $0.5 \mathrm{mag}$. in his own observations of RY Sgr, covering 1920 to 1932, and concluded that the star was pulsating. A photometric and spectroscopic survey of RY Sgr (Alexander et a1 1972) confirmed that the star was a pulsating variable. A mean period of 38.6 days was found in the photometric and radial velocity curves.

A series of high resolution spectra of RY Sgr, obtained by Cottrel1 and Lambert (1982), revealed absorption line splitting at certain phases. The line split spectra were obtained 80.8 days apart. The separation suggests a correlation between the line splitting and the 38.6 day pulsation period reported by Alexander et al. The relationship was not investigated due to a lack of data.

\section{RESULTS}

A continuing series of high resolution échelle spectra and accompanying photometry, initiated in 1983 (Lawson 1985), have been obtained with the two $61 \mathrm{~cm}$ reflectors at Mount John University Observatory. An initial series of spectra, completed in June 1984, isolated the line splitting event and established an ephemeris to aid future observations. The spectral range chosen was $5000 \AA$ to $7000 \AA$. From August to November 1984 (JD 2445924-6007) spectra and photometry were obtained as regular1y as possible to determine the nature of the radial velocity variations 
and correlate the line splitting event. A plate showing line splitting was obtained on JD 2445971. The light and radial velocity curves are reproduced in Figure 1. The velocity reversal occurs as the star contracts to minimum radius and then re-expands.

Many strong absorption lines show splitting. Most of the split lines are C I and ionised species, for example; Ba II, Fe II, Si II and Ti II. Weak lines ( $10 \%$ to $15 \%$ central depth) do not appear split. These observations reflect comments made by Danziger (1963) about line split and line broadened spectra obtained by him in 1961. Danziger found many strong ionised lines were split and no obvious examples of split weak lines. He did not detect any split neutral 1ines. In contrast, Cottre11 and Lambert (1982) found most, if not a11, 1ines displayed splitting although the companion lines were often very weak. The ability to detect the weak companion lines is probably related to the superior signal-to-noise capability of the Reticon system employed by Cottrell and Lambert compared to the image intensifier/photographic plate system used in this programme.

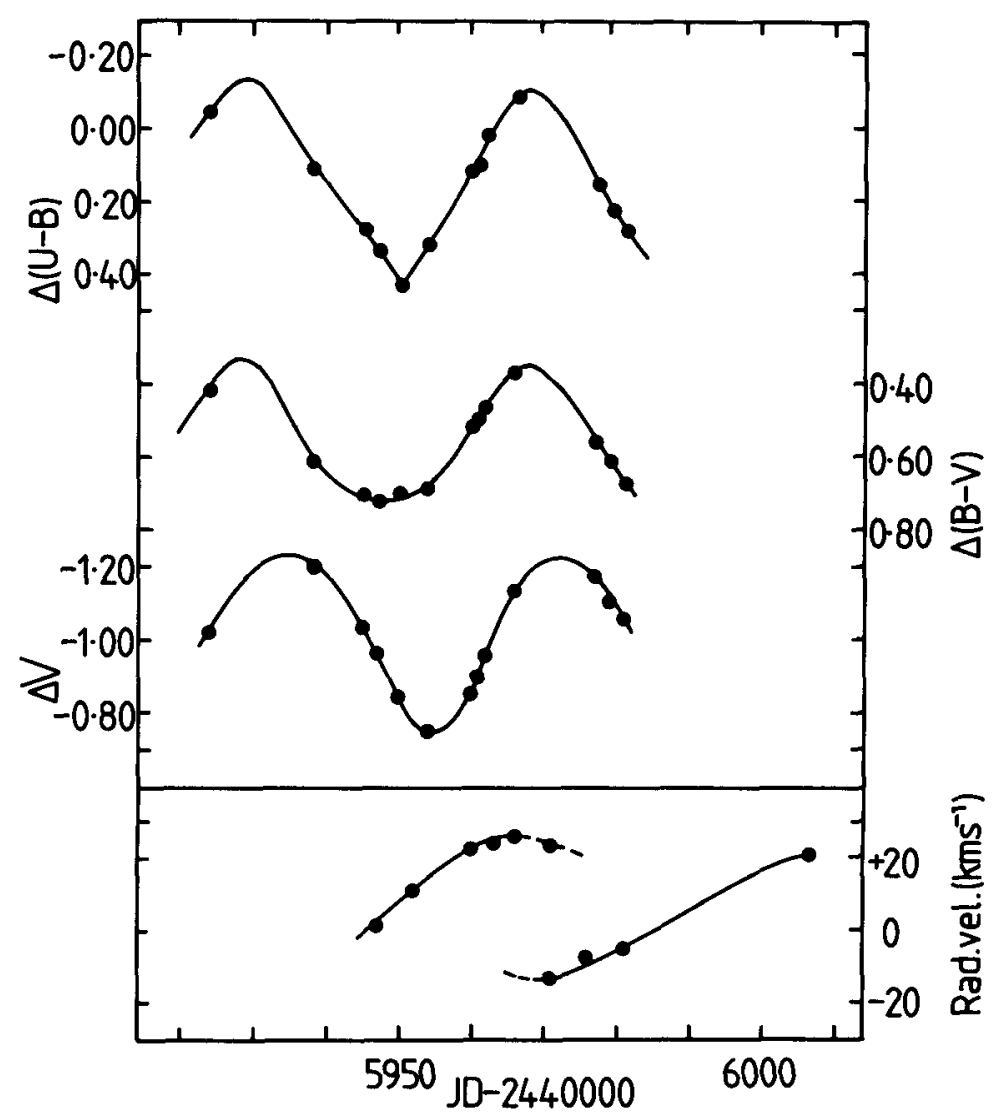

Figure 1. Photometric and radial velocity curves plotted against JD. The velocity split occurs at JD 2445971. 


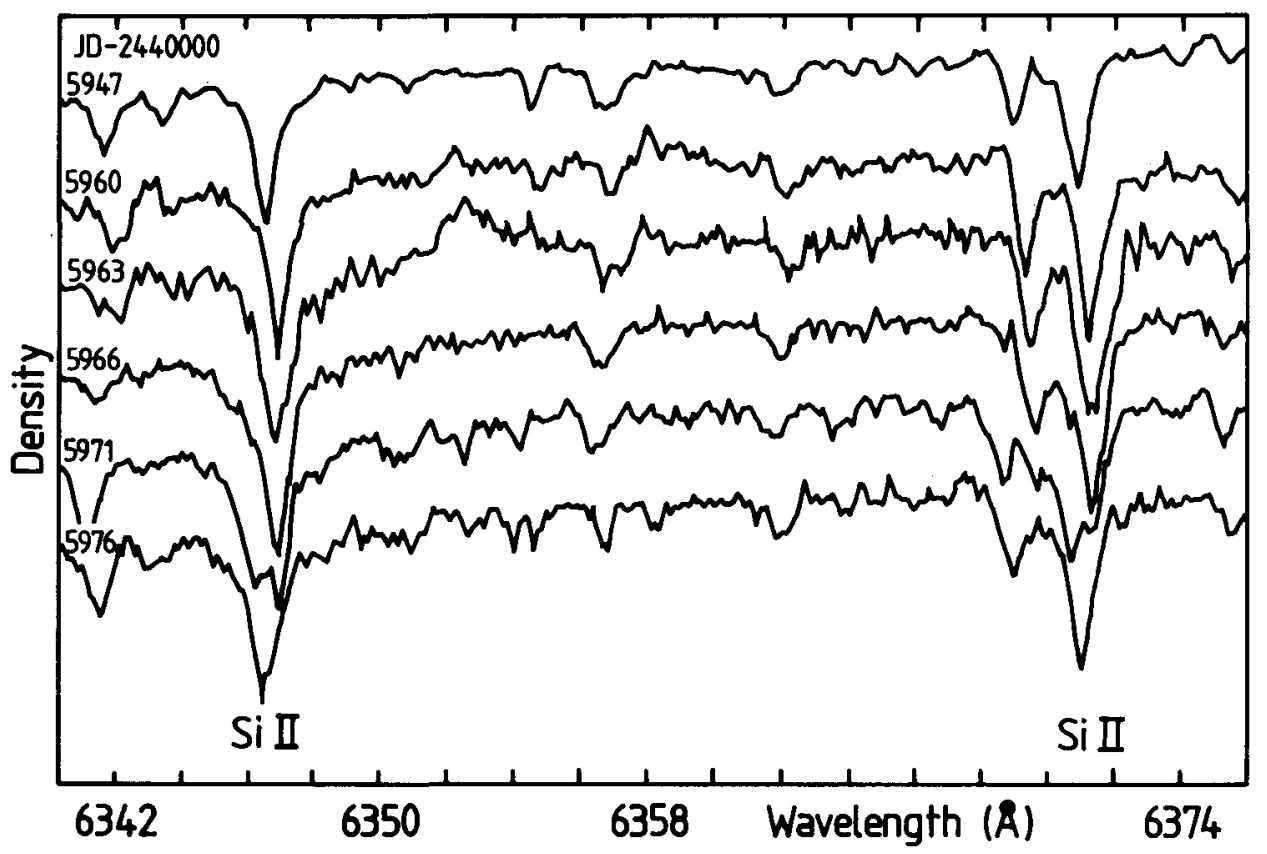

Figure 2. The evolution of the $6347 \AA$ and $6371 \AA$ Si II lines throughout the pulsation cycle.

The other notable variation seen in the absorption line spectra throughout the pulsation cycle is the general washing out of the spectrum approaching the line splitting event. This effect is especially noticeable in weak lines, many lines becoming almost indistinguishable from the continuum. The spectrum reverts to its normal form after the line splitting event and remains essentially unchcnged throughout the remainder of the pulsation cycle.

Spectra obtained 5 days prior, and 5 days after the line split spectra show little indication of the event other than minor asymmetric line profiles. This indicates the time scale of the event is about 10 days ( 0.23 of a period). The evolution of the $6347 \AA$ and $6371 \AA \mathrm{Si}$ II lines throughout the pulsation cycle is illustrated in Figure 2.

The line splitting event has been interpreted as a shock wave appearing about the phase of minimum radius. This result is broadly consistent with Saio and Wheeler's (1985) models for $M<1 M_{0}$, $T_{\text {eff }}=$ $7000 \mathrm{~K}$ pulsating $\mathrm{R} \mathrm{CrB}$ stars.

\section{DISCUSSION}

The radial velocity reversal is more rapid than indicated in the radial velocity analysis of RY Sgr by Alexander et al (1972). Our data suggests the reversal occurs within 5 days ( phase $=0.12$ ) whereas the data of Alexander et al indicates the reversal occurs in a mean of 19 days ( phase $=0.50$ ). It is possible, because the low resolution spectra of Alexander et al failed to reveal line splitting, that the radial 


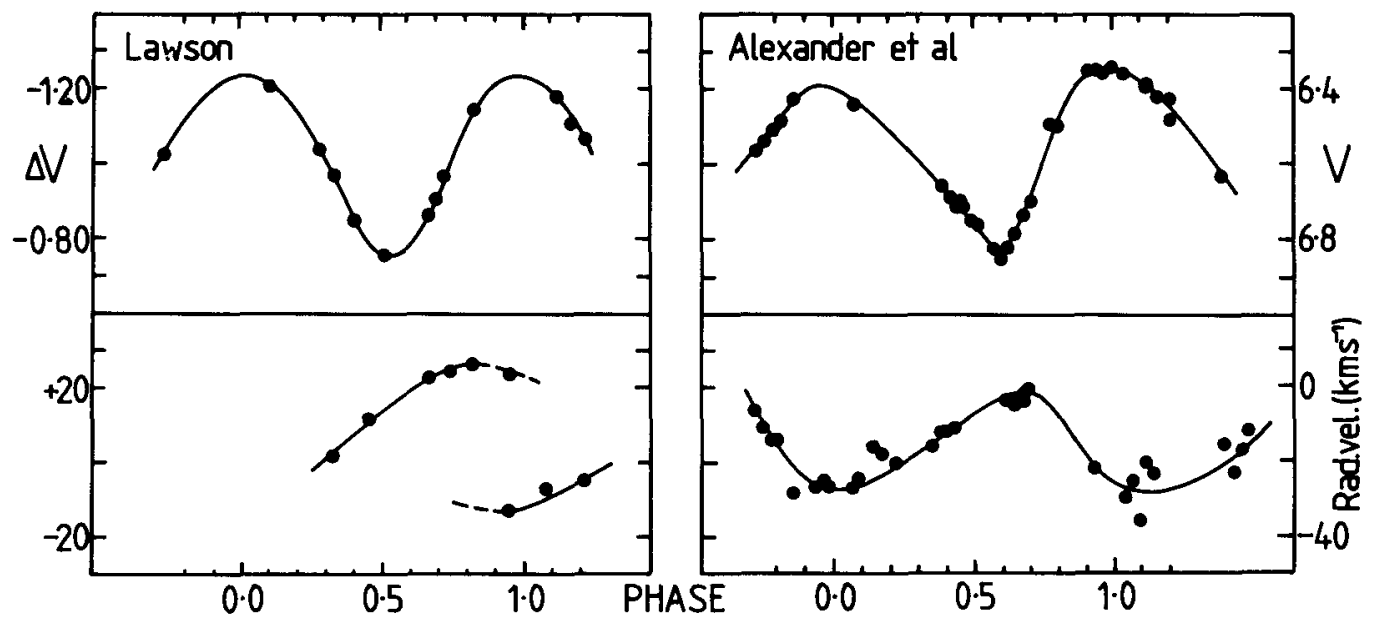

Figure 3. A comparison between the light and radial velocity curves of Alexander et al and our programme. The data of Alexander et al were obtained between JD 2440740 and JD 2440800 .

velocity curve has been smoothed by averaging velocity measurements during the line splitting event. The light and radial velocity curves of Alexander et al and our programme are compared in Figure 3.

Pugach (1977) has found the beginnings of declines to obscurational minima are related to the phase of pulsation. The declines begin at, or just prior to, Vmax. Since the phase of Vmax is comparable to the phase of the line splitting event (see Figure 3), a correlation between the declines and the formation of the line splitting event appears likely.

\section{CURRENT RESEARCH}

Recent work has included continued photometric coverage and attempts to investigate the line splitting event in greater detail. Spectra obtained in June 1985 (JD 2446243-47) cover the decline of the event. Spectra covering the formation of the event are required to satisfactorily analyse the phenomenon.

\section{REFERENCES}

Alexander, J.B., Andrews, P.J., Catchpole, R.M., Feast, M.W.,

Lloyd Evans, T., Menzies, J.W., Wisse, P.N.J. and Wisse, M., 1972, M.N.R.A.S., 158, 305.

Cottre11, P.L. and Lambert, D.L., 1982, Obs., 102, 149.

Danziger, I.J., 1963, thesis, Australian Nat. Üniv.

Jacchia, L., 1933, P. Oss. Astr. U. Bol., 2, 173.

Lawson, W.A., 1985, thesis, Univ. of Canter bury.

Pugach, A.F., 1977, Info. Bu11. Variable Stars, No. 1277.

Saio, H. and Wheeler, J.C., 1985, Ap. J., 295, 38. 


\section{DISCUSSION}

WING: Your radial velocity curve for RY Sgr looks very similar to the one Abt obtained many years ago for the RV Tauri star U Mon, although I believe the splitting in $U$ Mon was detected for a larger fraction of the cycle.

LAWSON: The line splitting in $U$ Mon was detected for about half a period. Observations of shock waves in pulsating stars are not uncommon. W Virginis stars, for example, show line splitting. Saio and Wheeler have shown for $R \mathrm{CrB}$ stars that a shock is essential if the pulsation amplitude is to remain bound. Perhaps this is also true for other classes of pulsators.

N.K. RAO: Did I understand you said that there is line doubling at certain phases in $\mathrm{R}$ CrB?

LAWSON: There is perhaps an indication of line asymmetry in some $\mathrm{R} \mathrm{CrB}$ spectra. More spectra are required.

LYNAS-GRAY: What is the maximum line-splitting observed in RY Sgr?

LAWSON: The degree of line splitting is $0.4 \AA$ to $0.6 \AA$. 